\title{
Spontaneous fluxon formation in annular Josephson tunnel junctions
}

\author{
Monaco, R.; Mygind, Jesper; Rivers, R.
}

Published in:

Physical Review B Condensed Matter

Link to article, DOI:

10.1103/PhysRevB.67.104506

Publication date:

2003

Document Version

Publisher's PDF, also known as Version of record

Link back to DTU Orbit

Citation (APA):

Monaco, R., Mygind, J., \& Rivers, R. (2003). Spontaneous fluxon formation in annular Josephson tunnel junctions. Physical Review B Condensed Matter, 67(10), 104506. https://doi.org/10.1103/PhysRevB.67.104506

\section{General rights}

Copyright and moral rights for the publications made accessible in the public portal are retained by the authors and/or other copyright owners and it is a condition of accessing publications that users recognise and abide by the legal requirements associated with these rights.

- Users may download and print one copy of any publication from the public portal for the purpose of private study or research.

- You may not further distribute the material or use it for any profit-making activity or commercial gain

- You may freely distribute the URL identifying the publication in the public portal 


\title{
Spontaneous fluxon formation in annular Josephson tunnel junctions
}

\author{
R. Monaco, ${ }^{1, *}$ J. Mygind, ${ }^{2, \dagger}$ and R. J. Rivers ${ }^{3, \$}$ \\ ${ }^{1}$ Istituto di Cibernetica del CNR, I-80078 Pozzuoli, Italy \\ and Unita' INFM-Dipartimento di Fisica, Universita' di Salerno, I-84081 Baronissi (SA), Italy \\ ${ }^{2}$ Department of Physics, Technical University of Denmark, B309, DK-2800 Lyngby, Denmark \\ ${ }^{3}$ Centre of Theoretical Physics, University of Sussex, Brighton, BN1 9QJ, United Kingdom
}

(Received 23 July 2002; published 13 March 2003)

\begin{abstract}
It has been argued by Zurek and Kibble that the likelihood of producing defects in a continuous phase transition depends in a characteristic way on the quench rate. In this paper we discuss our experiment for measuring the Zurek-Kibble (ZK) scaling exponent $\sigma$ for the production of fluxons in annular symmetric Josephson tunnel junctions. The predicted exponent is $\sigma=0.25$, and we find $\sigma=0.27 \pm 0.05$. Further, there is agreement with the ZK prediction for the overall normalization.
\end{abstract}

DOI: 10.1103/PhysRevB.67.104506

PACS number(s): 67.40.Vs, 11.27.+d, 05.70.Fh, 11.10.Wx

\section{INTRODUCTION}

As the early universe cooled it is believed to have undergone a series of spontaneous phase transitions, whose inhomogeneities (monopoles, cosmic strings, domain walls) have observable consequences, for structure formation in particular. These defects appear because the correlation length $\xi$ of the field (or fields) whose expectation value is the order parameter is necessarily finite for a transition that is implemented in a finite time, whether it be continuous or not.

It is difficult to determine the microscopic dynamics of such fields but, using only simple causal arguments, Kibble $^{1,2}$ made estimates of this early field ordering, and the density of topological defects produced at grand unified theory transitions at $10^{-35} \mathrm{~s}$. Unfortunately, because the nature of the field theories is not known with any reliability, and the effects of their evolution are not visible until the decoupling of the radiation and matter $10^{6}$ years later, it is impossible to provide unambiguous checks of these predictions. However, causality is such a fundamental notion that Zurek suggested ${ }^{3,4}$ that identical causal arguments, with similar predictions, were applicable to condensed-matter systems for which direct experiments on defects could be performed. In addition to their intrinsic interest for a better understanding of the dynamics of transitions in condensed matter, the hope is that successful tests of these predictions can lead to a better understanding of phase transitions in quantum fields.

Several experiments in condensed matter systems have already been performed ${ }^{5-11}$ to test the Zurek-Kibble predictions, with mixed results. It is these predictions that we have tested here, using annular Josephson tunnel junctions, for which the defects are fluxons.

This paper is organized as follows. In the following section we discuss the Zurek-Kibble (ZK) scenario. Other sections then give the predictions for annular Josephson tunnel junctions (AJTJ's), show how the fluxons are measured, the nature of the samples, describe the experimental setup and, finally, present the measurements and their agreement with the predictions. An abbreviated description of the experiment has been given elsewhere. ${ }^{12}$ Details of the theoretical analysis can be found in Refs. 13 and 14, in which an earlier experiment ${ }^{15}$ by two of us (R.M. and J.M.) was analyzed to demonstrate its compatibility with the $\mathrm{ZK}$ analysis, even though it had not been performed with this in mind.

\section{ZUREK-KIBBLE CAUSALITY}

Consider a system with critical temperature $T_{c}$, cooled through that temperature so that, if $T(t)$ is the temperature at time $t$, then $T(0)=T_{c} . \dot{T}(0)=-T_{c} / \tau_{Q}$ defines the quench time $\tau_{Q}$.

There are several ways ${ }^{4}$ of formulating the Zurek-Kibble causality bounds, but they all depend on the fact that, as the transition begins to be implemented, there is a maximum speed $c(t)=c(T(t))$ at which the system can become ordered. For relativistic quantum-field theory $c$ is the speed of light. For superfluids, $c(t)$ is the speed of second sound, vanishing at $t=0$. For Josephson tunnel junctions $c(t)$, which depends on the nature of the junction, is the Swihart ${ }^{16}$ velocity.

Suppose that the equilibrium (adiabatic) correlation length $\xi_{a d}(t)=\xi_{a d}(T(t))$ diverges near $t=0$ as

$$
\xi_{a d}(t)=\xi_{0}\left|\frac{t}{\tau_{Q}}\right|^{-\nu}
$$

However, the true nonequilibrium correlation length $\xi(t)$ can only change so much in a finite time, and does not diverge. Kibble and Zurek made two assumptions.

(1) First, the correlation length $\bar{\xi}$ of the fields that characterizes the onset of order is the equilibrium correlation length $\bar{\xi}=\xi_{a d}(\bar{t})$ at some appropriate time $\bar{t}$.

(2) Second, we can measure $\bar{\xi}$ experimentally by measuring the number of defects, assuming that the defect separation $\xi_{d e f}=O(\bar{\xi})$.

There are several ways to estimate $\bar{t}$ (the "causal time"), explicit or implicit in the early work of Zurek. ${ }^{4}$ Most simply we have the following.

(1) $\xi(t)$ cannot grow faster than $c(t)$. This is true both before and after the transition. That is, $\bar{t}$ is defined by the condition that $\dot{\xi}_{a d}(\bar{t}) \approx-c(\bar{t})$. 
(2) The relaxation time for the long-wavelength modes $\tau(t)$ is defined by $c(t)=\xi_{a d}(t) / \tau(t)$. From this viewpoint, $\bar{t}$ is that time when we can return to an adiabatic regime, $\bar{t}$ $\approx \tau(\bar{t})$.

In simple systems these estimates agree up to numerical factors approximately unity. ${ }^{4}$ They give $\bar{t}$ of the form $\bar{t}$ $\sim \tau_{Q}^{1-\gamma} \tau_{0}^{\gamma}$, where $\tau_{0} \ll \tau_{Q}$ is the cold relaxation time of the longest wavelength modes, and the critical exponent $\gamma$ depends on the system. As a result, $\tau_{Q} \gg \bar{t} \gg \tau_{0}$. Identifying the initial domain size and defect separation as in the second $\mathrm{ZK}$ assumption then gives

$$
\bar{\xi} \sim \xi_{a d}(\bar{t})=\xi_{0}\left(\frac{\tau_{Q}}{\tau_{0}}\right)^{\sigma} \gg \xi_{0}
$$

where $\sigma=\gamma \nu$. This is very large on the scale of cold defects that shrink to size $\xi_{a d}\left(T_{\text {fin }}\right)=O\left(\xi_{0}\right)$, where $T_{\text {fin }}$ is the final temperature and $\xi_{0}$ is determined from the microscopic dynamics. We term $\sigma$ the ZK scaling index.

The analysis above is for large systems, of linear size $L$ $\gg \bar{\xi}$. For the experiment that we shall describe below, of AJTJ's of circumference $C$, we find that $C<\bar{\xi}$. In fact, we expect the conclusions to be equally valid for small systems, for which the relevant time might, incorrectly, seem to be the time when the coherence length becomes smaller than the system. The reason is that the causal bounds (1) are to be thought of as a shorthand for the underlying dynamics. At the microscopic level, causality along the lines above is not explicit, although encoded in the relevant dynamical equations. The picture is rather one of order being established through the growth of the amplitudes of long-wavelength instabilities. The earliest time at which we can identify defects from this viewpoint is when the order parameters have achieved their equilibrium magnitudes. Qualitatively, for simple models this time is in good agreement with the causal time $\bar{t}$ above. There is no real surprise in this. It has been shown by one of us ${ }^{17,18}$ that, in general, the causal time and distance scales $\bar{t}$ and $\bar{\xi}=\xi(\bar{t})$ are just as we would expect from a dimensional analysis (in the mean-field approximation) and unstable modes grow exponentially, whereby the dependence of the causal time (and corresponding defect density) on the microscopic parameters is only logarithmic. In the same way, the distance between field zeros has the same scaling dependence on $\tau_{Q}$ as $\bar{\xi}$ of Eq. (1), up to logarithms.

As for the production of defects, there are transitional regions between different system ground states. For superfluids such as ${ }^{4} \mathrm{He}$ and superconductors these transitional regions are flagged by zeros of the scalar order-parameter field. For the case of JTJ's we shall see that this is generalized to zeros $(\bmod 2 \pi)$ of the order-parameter field. Any field crossing zero $(\bmod 2 \pi)$ has the potential to mature into a defect. However, only when the transition is complete will the field configurations in the vicinity of the zeros have the energy profile of a classical defect, the solution to the classical field equations. Thus, before the causal time, we now have a picture in which there is a fractal thermal fuzz of potential defects, whose density depends on the scale at which we look.
By the causal time some of these have developed into the (scale-independent) defects that we see subsequently (see Refs. 17 and 18). Because, from this viewpoint, we have many protodefects jockeying to become the real thing, the relevant scale to compare to the system size is not $\bar{\xi}$ but $\xi_{0}$, as before. Equivalently, counting field zeros $(\bmod 2 \pi$, or not) depends on the short-distance behavior of the field correlations. We do not expect a problem as long as $C \gg \xi_{0}$, as is always the case.

We conclude by noting that this is a very different picture from that of domains freezing in as the transition is approached from above $(t<0)$, which is how causal bounds were originally posed. ${ }^{2,3}$ What matters is that all these causal descriptions give results with the correct engineering dimensions. With this in mind, we keep the causal bound (1) as a convenient mnemonic.

\section{THE ZK PREDICTIONS FOR FLUXONS}

The order parameter of a Josephson tunnel junction at temperature $T<T_{c}$ is the phase difference $\phi$ of the macroscopic superconducting quantum-mechanical wave functions across the barrier. Using a Lagrangian formalism, GrønbechJensen et al. ${ }^{19}$ have shown that, for an annular JTJ with a distributed bias current $\Gamma, \phi$ obeys to the following perturbed Sine-Gordon equation:

$$
\frac{\partial^{2} \phi}{\partial x^{2}}-\frac{1}{c^{2}(T)} \frac{\partial^{2} \phi}{\partial t^{2}}-\frac{1}{\lambda_{J}^{2}(T)} \sin \phi=\Gamma+\frac{\alpha}{c^{2}(T)} \frac{\partial \phi}{\partial t}-\beta \frac{\partial^{3} \phi}{\partial x^{2} \partial t}
$$

provided the width $\Delta r$ of the annulus, of radius $r$, satisfies $\Delta r \ll r$ and $\Delta r \ll \lambda_{J}(T)$, the Josephson coherence length. In this case $x$ measures the distance along the annulus, and $c(T)$ is the Swihart velocity. $\alpha$ and $\beta$ are the coefficients of the losses due to the tunneling current and due to the surface impedance, respectively.

The boundary conditions for Eq. (2) are periodic ${ }^{20}$ and derive from fluxoid quantization: ${ }^{21} \phi(x+C)=\phi(x)+2 \pi n$, where $C=2 \pi r$ is the circumference of the junction and the winding number $n$ is an integer corresponding to the algebraic sum of fluxons trapped in the junction barrier at the normal-superconducting $(N-S)$ transition; $n$ is a topological system constant, that is, only fluxon-antifluxon $(F \bar{F})$ pairs can be created or annihilated as long as the junction remains in the superconducting state.

The classical fluxons are the "kinks" of the Sine-Gordon theory. As with other models of defect formation, Eq. (2) is only valid once the transition is complete: therefore, we shall not use it to study the appearance of fluxons. However, it is sufficient to enable us, in the spirit of the Zurek-Kibble scenario, to identify $\lambda_{J}(T)$, diverging at $T_{c}$, as the equilibrium correlation length $\xi_{a d}(T)$ to be constrained by causality. Further, the Swihart velocity $c(T)$ (with critical slowing down at $T=T_{c}$ ) measures the maximum speed at which the order parameter can change. ${ }^{16,22}$

A detailed discussion of the $\mathrm{ZK}$ bounds has been given elsewhere by us, ${ }^{13,14}$ and we refer the reader to these papers for more details. The JTJ's in our experiment are symmetric, 
by which is meant that the electrodes are made of identical superconducting material with the same energy gaps and the same $T_{c}$. For such JTJ's $\gamma=\nu=1 / 2{ }^{13,14}$ Therefore, at the time of their formation

$$
\bar{t}=\sqrt{\tau_{Q} \tau_{0}},
$$

the separation of fluxons is expected, in the ZK picture, to be

$$
\bar{\xi} \sim \xi_{0}\left(\frac{\tau_{Q}}{\tau_{0}}\right)^{1 / 4}
$$

where $\xi_{0}$ and $\tau_{0}$ have the same meaning as before. It is the prediction (3) that will be tested in our experiment.

In terms of the parameters of the JTJ's, $\xi_{0}$ has been inferred $^{14}$ as

$$
\xi_{0}=\sqrt{\frac{\hbar}{2 e \mu_{0} d_{s} a J_{c}(0)}},
$$

where $J_{c}(T)$ is the Josephson current density at temperature $T$. The parameter $a$ is given in terms of the superconductor gap energy and critical temperature and has a value between 3 and 5. If the thickness of the two superconducting electrodes differs, the effective thickness $d_{s}$ is the harmonic mean of the individual thicknesses. ${ }^{22}$ As for $\tau_{0}$, it is given as $\tau_{0}=\xi_{0} / c_{0}$, where $c_{0}$ defines the behavior $c(t)=c_{0}\left(t / \tau_{Q}\right)^{1 / 2}$ of the Swihart velocity for the system near $T=T_{c}$.

\section{MEASURING FLUXONS}

Once fluxons have appeared, Eq. (2) is relevant. A consequence of the periodic boundary conditions in AJTJ's is that fluxons behave as relativistic particles on an infinite lossy line. In the absence of any current through the barrier and/or externally applied magnetic field, the fluxons experience a flat potential and therefore are in indifferent equilibrium as far as the barrier is homogeneous and pin hole free; in the reality, the barrier defects act as small pinning or repulsive potentials for the fluxons. Due to the losses, after the transient regime is over the fluxons are still. Unfortunately static fluxons are difficult to reveal, since according to the second Josephson law, any static phase profile $\phi_{t}(x, t)=0$ does not alter the junction zero-voltage state that is also typical of a flat profile $\phi(x, t)=$ const, corresponding to the absence of any trapped fluxon.

In contrast, whenever fluxons travel around an AJTJ they leave a clear signature on the junction current-voltage characteristic $(\mathrm{CVC})$ and therefore are easily detectable. In fact, as soon as a bias current is fed to the AJTJ, the fluxons move as magnetic dipoles under the action of the resulting Lorentz force. The fluxon dynamics in long JTJ's is a well-known topic and has received a great deal of both theoretical and experimental attention in the last few decades. If the external bias is assumed to be uniform over the junction area, then, as a result of the balance between the externally supplied power and the internally dissipated power, the fluxons move with a constant speed: the larger the external bias, the greater the fluxon speed, but never exceeding its relativistic limit sets by the Swihart velocity. The motion direction depends both on the current sign and fluxon polarity (i.e., whether $n= \pm 1$ ) for a given bias current. Fluxons having different polarities travel in opposite directions and are likely to annihilate when they collide at low speed.

Quantitatively, if a fluxon travels around an AJTJ having a mean circumference $C$ with a constant speed $v$, then it has angular speed $\omega=2 \pi v / C$ and the phase $\phi$ advances of $2 \pi$ each period $T=2 \pi / \omega=C / v$. Therefore, according to the second Josephson equation, an average voltage $V$ develops across the junction equal to $V=\Phi_{0} / 2 \pi\langle d \phi / d t\rangle=\Phi_{0} / T$ $=\Phi_{0} v / C$, which is proportional to the fluxon velocity. In other words, the presence of a traveling fluxon sets the junction in a finite voltage state than can be easily measured on its CVC.

By also changing the bias current through the barrier the voltage drop changes and a new branch called zero-field step (ZFS) appears on the junction CVC; the ZFS represents the relation between the applied Lorentz force (proportional to the bias current) and the fluxon speed (proportional to the voltage). When $N$ fluxons travel around an AJTJ, the last expression is easily generalized to give a junction voltage $V=N \Phi_{0} C / v$. In the last expression, $N$ is the total number of traveling fluxons and can be larger than the winding number $n$ if $F \bar{F}$ pairs are traveling around the annulus. Therefore, we count the number of traveling fluxons by simply measuring the voltage across the AJTJ.

These properties makes AJTJ's very competitive with respect to other solid-state systems proposed to test the ZurekKibble mechanism. Our idea is to perform a large number of $\mathrm{N}-\mathrm{S}$ transitions on the same AJTJ with no external current or magnetic field; at the end of each cycle, the possible spontaneously generated fluxons are static. Then we supply an external current that sets the fluxons (if any) in motion around the annulus and measure the number of traveling fluxons by a careful inspection of the junction CVC. Due to the annihilation of a fluxon-antifluxon pair, this idea works well as long as the chances to spontaneously generate two fluxons are small.

Figs. 1(a), (b), and (c) represent the CVC of the same AJTJ with no fluxon trapped, with one fluxon trapped, and with two fluxons trapped, respectively. We note that with no trapped fluxons the zero-voltage current is very large and only $F \bar{F}$ current steps appear at finite voltage. In the other two cases the supercurrent is rather small (theoretically it should be vanishingly small in ideal, pin-hole-free barriers) and large current branches can be observed at finite voltages corresponding to the fluxons and, possibly, $F \bar{F}$ pairs traveling around the junction.

\section{THE SAMPLES}

High quality $\mathrm{Nb} / \mathrm{Al}^{-\mathrm{Al}_{o x}} / \mathrm{Nb} \mathrm{JTJ}$ 's were fabricated on 0.5 $\mathrm{mm}$-thick silicon substrates using the trilayer technique in which the junction is realized in the window opened in a $\mathrm{SiO}$ insulator layer. Details of the fabrication process can be found in Ref. 23 . On each $15 \times 24 \mathrm{~mm}^{2}$ chip four JTJ's were integrated, of which three ring-shaped junctions having a 

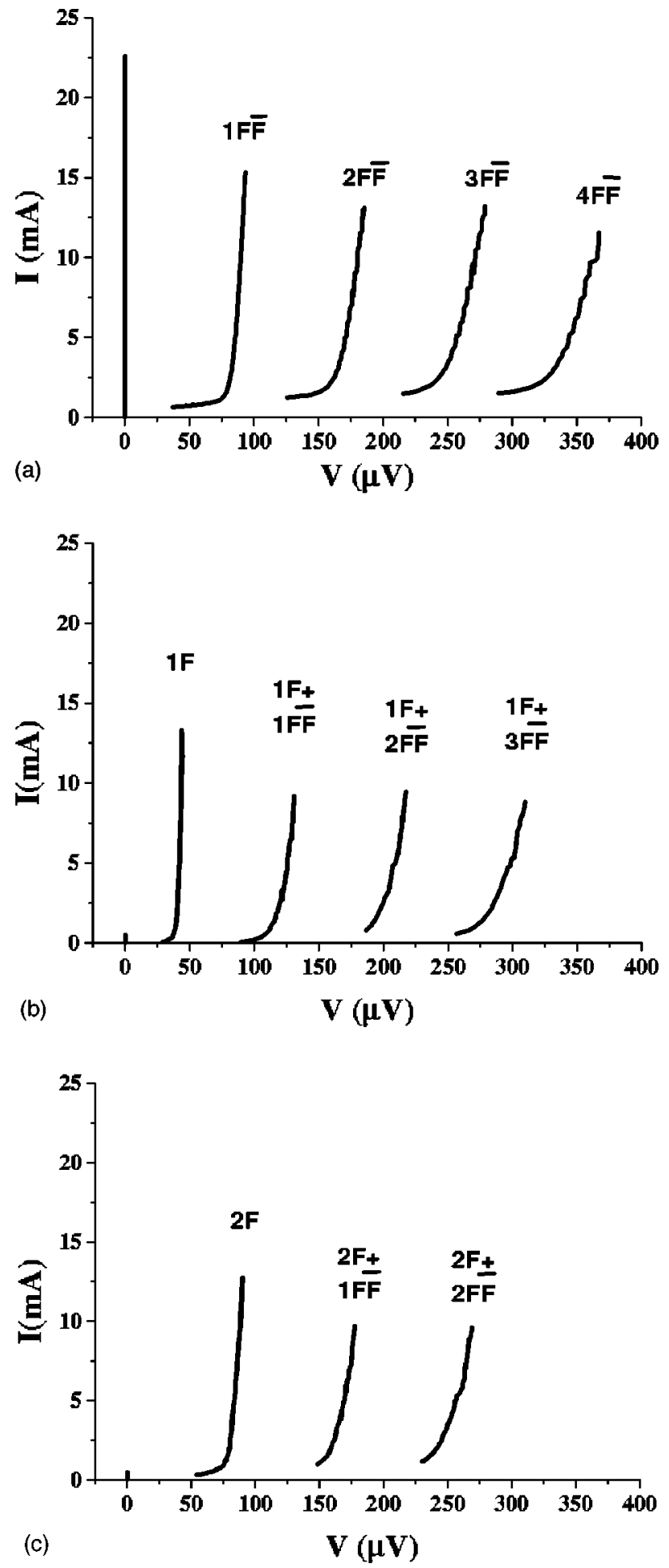

FIG. 1. Low-voltage part of the experimentally measured current-voltage characteristics of the same annular Josephson tunnel junction (a) without trapped fluxons, (b) with one trapped fluxon, and (c) with two trapped fluxons. For each current branch the corresponding number of traveling fluxons $F$ and fluxon-antifluxon pairs $F \bar{F}$ is indicated.

mean circumference $C=500 \mu \mathrm{m}$ and a width $\Delta r=4 \mu \mathrm{m}$ and one $4 \times 500 \mu \mathrm{m}^{2}$ overlap-type linear junction. The socalled "idle region," i.e., the overlapping of the wiring layer onto the base electrode was about $3 \mu \mathrm{m}$ for all the junctions.
TABLE I. Geometrical and electrical parameters of two selected annular Josephson tunnel junctions at $4.2 \mathrm{~K}$.

\begin{tabular}{lcc}
\hline \hline Sample & $A$ & $B$ \\
\hline Mean circumference $C(\mu \mathrm{m})$ & 500 & 500 \\
Width $\Delta r(\mu \mathrm{m})$ & 4 & 4 \\
Zero field critical current $I_{o}(\mathrm{~mA})$ & 33 & 2.5 \\
Maximum critical current $I_{\max }(\mathrm{mA})$ & 39 & 2.7 \\
Gap quasiparticle current step $\Delta I_{g}(\mathrm{~mA})$ & 88 & 5.2 \\
$I_{\text {max }} / \Delta I_{g}$ & 0.45 & 0.52 \\
Critical current density $J_{c}\left(\mathrm{~A} / \mathrm{cm}^{2}\right)$ & 3050 & 180 \\
Josephson length $\lambda_{J}(\mu \mathrm{m})$ & 6.9 & 28 \\
Normalized mean circumference $C / \lambda_{J}$ & 72 & 18 \\
Quality factor $V_{m}(\mathrm{mV})$ & 49 & 63 \\
Normal resistance $R_{N}(\mathrm{~m} \Omega)$ & 36 & 610 \\
ZFS1 asymptotic voltage $(\mu \mathrm{V})$ & 51 & 53 \\
\hline \hline
\end{tabular}

The thicknesses of the base, top, and wiring layer were 200, 80 , and $400 \mathrm{~nm}$, respectively.

For all samples the high quality has been inferred by a measure of the $I-V$ characteristic at $T=4.2 \mathrm{~K}$. In fact, the subgap current $I_{s g}$ at $2 \mathrm{mV}$ was small compared to the current rise $\Delta I_{g}$ in the quasiparticle current at the gap voltage $V_{g}$, typically $\Delta I_{g}>35 I_{s g}$, the gap voltage was as large as $V_{g}=2.76 \mathrm{mV}$ and the maximum critical current $I_{c}$ was larger than $0.55 \Delta I_{g}$ for the overlap-type junction. Furthermore, the application of a strong enough external magnetic field in the barrier plane completely suppressed any Josephson structures indicating the absence of electrical shorts in the barrier. It is important to mention that (i) no logarithmic singularity has been observed in the CVC's at low voltages and (ii) the temperature dependence of the critical current was linear as the temperature $T$ approached the critical temperature $T_{C}$; both these observations assure us that the junctions are symmetric, i.e., no detectable difference can be assumed between both the energy gaps $\Delta_{1,2}$ and the critical temperatures $T_{C 1,2}$ of the junction electrodes 1 and 2 in the proximity of the barrier. The maximum Josephson current density $J_{J}$ was of the order of $1 \mathrm{kA} / \mathrm{cm}^{2}$ corresponding to a specific barrier normal resistance $\rho_{N}$ of about $200 \Omega \mu \mathrm{m}^{2}$.

Many samples have been measured. For clarity only two will be discussed here. The geometrical and electrical (at 4.2 $\mathrm{K})$ parameters of the two selected annular junctions on different wafers are listed in Table I. They have the same geometry (both the base and top electrode have a hole concentric to the ring), but differ in the critical current densities, i.e., in the normalized mean circumferences $C / \lambda_{J}$. The critical current density has been calculated from the measured quasiparticle current step, $\Delta I_{g}$, at the gap voltage. The values of the barrier magnetic thickness $\Lambda=180 \mathrm{~nm}$ has been used for numeric calculations. On each same chip a linear overlaptype junction with the same width, length, and idle region was used in order to measure the junction Swihart velocity $c_{0}$ with a geometry in which the effects of the self-field are minimized. The value of $c_{0}=1.4 \times 10^{7} \mathrm{~m} / \mathrm{sec}$, due to the effect of the idle region, is 1.5 times larger than that expected 
To the vacuurI purrip

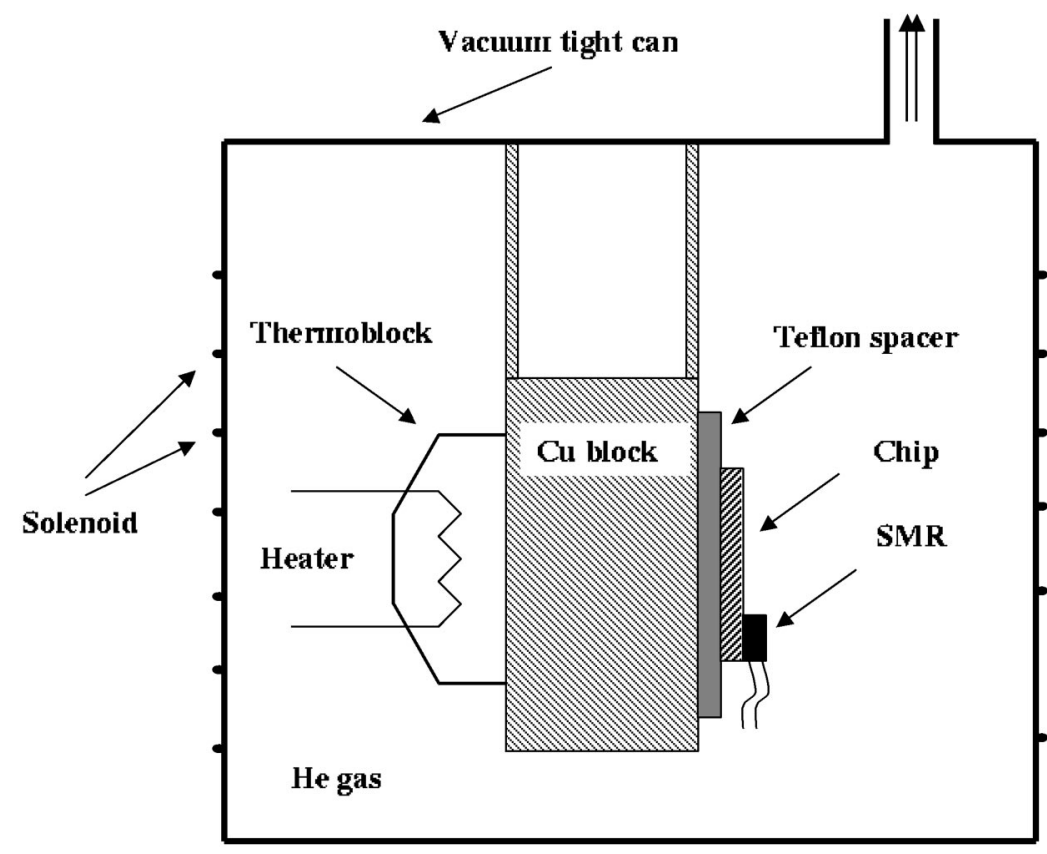

FIG. 2. Sketch (dimensions are not to scale) of the cryogenic insert developed to perform the junction thermal cycles with a time scale changing over a broad range.

\section{LHe bath}

for a bare junction. This value of $c_{0}$ corresponds to a value of $0.08 \mathrm{~F} / \mathrm{m}^{2}$ for the barrier effective specific capacitance.

The data in Table I show that both samples are highquality, long $\left(C \gg \lambda_{J}\right)$ annular JTJ's.

\section{THE EXPERIMENTAL SETUP}

In order to vary the quenching time in the broadest possible range, we have realized the experimental setup schematically shown in Fig. 2.

A massive $\mathrm{Cu}$ block held to the sample holder by two thin $\mathrm{Cu}$ arms was used to increase the system thermal capacity. The chip was mounted on one side of this block and thermally loosely coupled to it by means of a 1-mm-thick teflon sheet. On the other side of the $\mathrm{Cu}$ block, a thermoblock consisting of a 50- $\Omega$ carbon resistor and two thermometers in order to measure and to, if necessary, stabilize the $\mathrm{Cu}$ block temperature, was mounted in good thermal contact. Finally a small-sized $100-\Omega$ resistor, more precisely a surface mount resistor (SMR), was kept in good thermal contact with the chip by means of a small amount of vacuum grease.

This system, due to the two heating elements placed in tight and loose thermal contacts with the chip and with the proper choice of the thermal loads, allowed us to perform the sample quenching over two quite different time scales. In fact, by means of the resistor in the thermoblock, a long time scale was achieved by heating the chip through the $\mathrm{Cu}$ block and the teflon sheet; on the contrary, a short current pulse through the surface mount resistor on the chip attained much shorter thermal cycles.

These two completely different quenching techniques provide time scale ranges that do not overlap, leaving a gap between 0.2 and $1 \mathrm{~s}$, which would require a third quenching technique to be filled. We stress that just using a single sample holder with smaller heat capacity would not give us access to the same time scales. We could not get quench times as short as those from the SMR and, even with a very small exchange gas pressure, could not get times as long as those with the larger sample holder on using the mechanical pumps available to us.

The whole system was kept in a vacuum tight can immersed in the LHe bath at $\mathrm{He}$ gas pipeline pressure. The pressure of He gas inside the can could be varied in order to modify the heat exchange between the chip and the environment and, in turn, the speed of the sample cooling. A solenoid was wound around the can to provide a strong vertical magnetic field and Helmholtz coils were instead placed inside the can to generate a weak horizontal magnetic field in the barrier plane in order to tune the critical currents of the annular junctions to their maximum values.

The temperature dependence of the junction gap voltage was exploited to monitor the temperature of the junction itself during the thermal cycle. Figures 3(a) and (b) show the digitally measured $V_{g}(t)$ for sample $A$ current biased on the quasiparticle curve at $17.7 \mathrm{~mA}$, (i.e., at about one-fifth of $\Delta I_{g}$ at $T=4.2 \mathrm{~K}$ ), for a slow and a fast thermal cycle, respectively. In the case of Fig. 3(a), a 100-mA current was fed to the thermoblock heater for about $4 \mathrm{~s}$ in order to increase the junction temperature up to its critical value where the sample CVC becomes a straight line with a slope corresponding to the junction normal resistance $R_{N}$. In the case of Fig. 3(b) a 20-V-high and 4-ms-wide voltage pulse was applied at the SMR. In Fig. 3 the time origin is arbitrarily set at the instant we began to feed the heating elements. It is important to observe that the time scale in Fig. 3(a) is about 50 

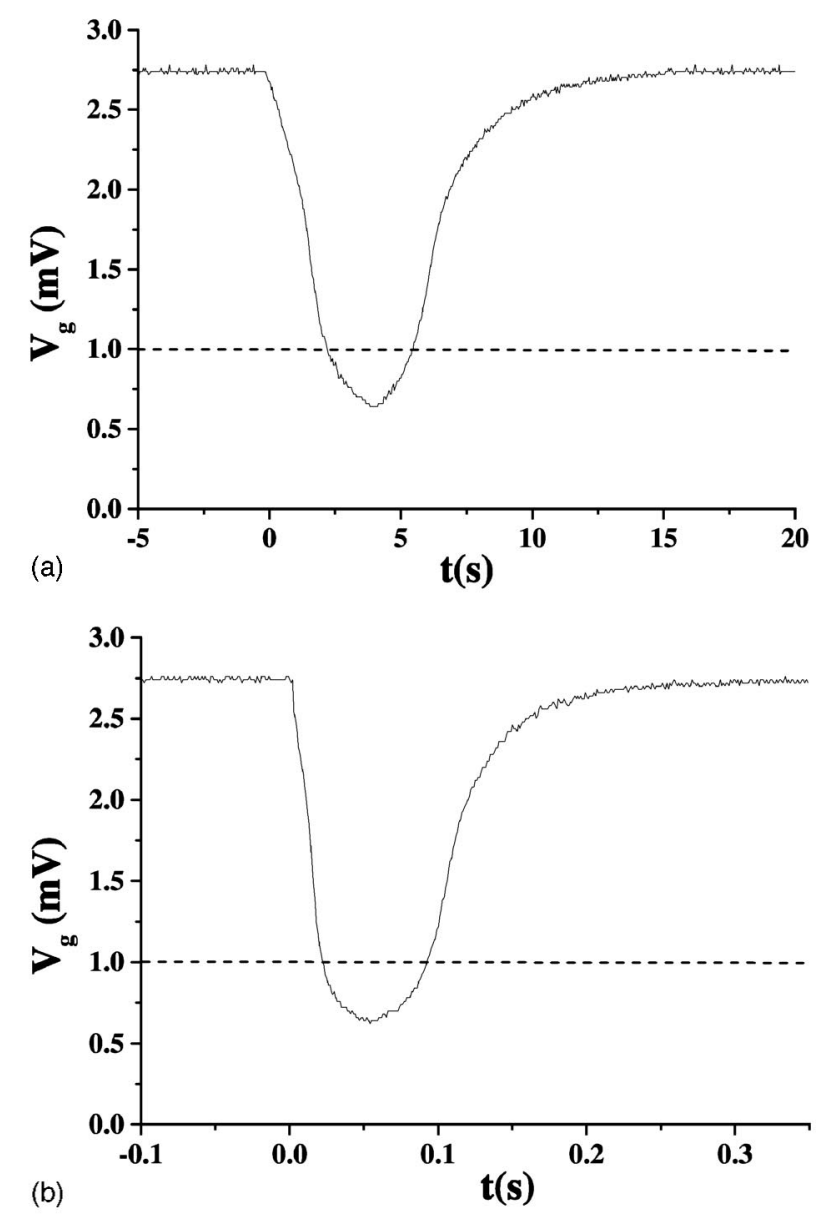

FIG. 3. Digitally measured time dependence of the junction gap voltage during the (a) "slow" and (b) "fast" thermal cycle. For these measurements the junction was biased at about 1/5 of the gap quasiparticle current step. The horizontal dashed lines indicate the voltage threshold above which Eq. (4) can be used to relate the junction voltage at its temperature.

times larger than that of Fig. 3(b), although the curve shapes are quite similar.

In our samples the current jump at the gap voltage is very steep and, at $T=4.2 \mathrm{~K}$, the voltage changes by less than $1 \%$ when the current is changed from $10 \%$ to $90 \%$ of the total current jump $\Delta I_{g}$ and by less of $10 \%$ at $T=8.5 \mathrm{~K}$. Furthermore, at $T=4.2$ and $8.5 \mathrm{~K}$ and for this bias current, the junction voltage was equal to 2.74 and $1.0 \mathrm{mV}$, respectively. Therefore, assuming that the electrode gap energies are equal $\Delta_{1}(T)=\Delta_{2}(T)=\Delta(T)$, and that in the $4.2-8.5 \mathrm{~K}$ range we can neglect the thermal gap smearing, the analytical expression found by Thouless ${ }^{24}$ for the gap energy in a strongcoupling superconductor

$$
\frac{\Delta(T)}{\Delta(0)}=\tanh \frac{\Delta(T)}{\Delta(0)} \frac{T_{c}}{T}
$$

also applies to the junction gap voltage that is proportional to it. The dashed lines in Fig. 3 indicate the voltage threshold above which Eq. (4) can be used to relate the junction voltage at its temperature, without any significant loss of accu-
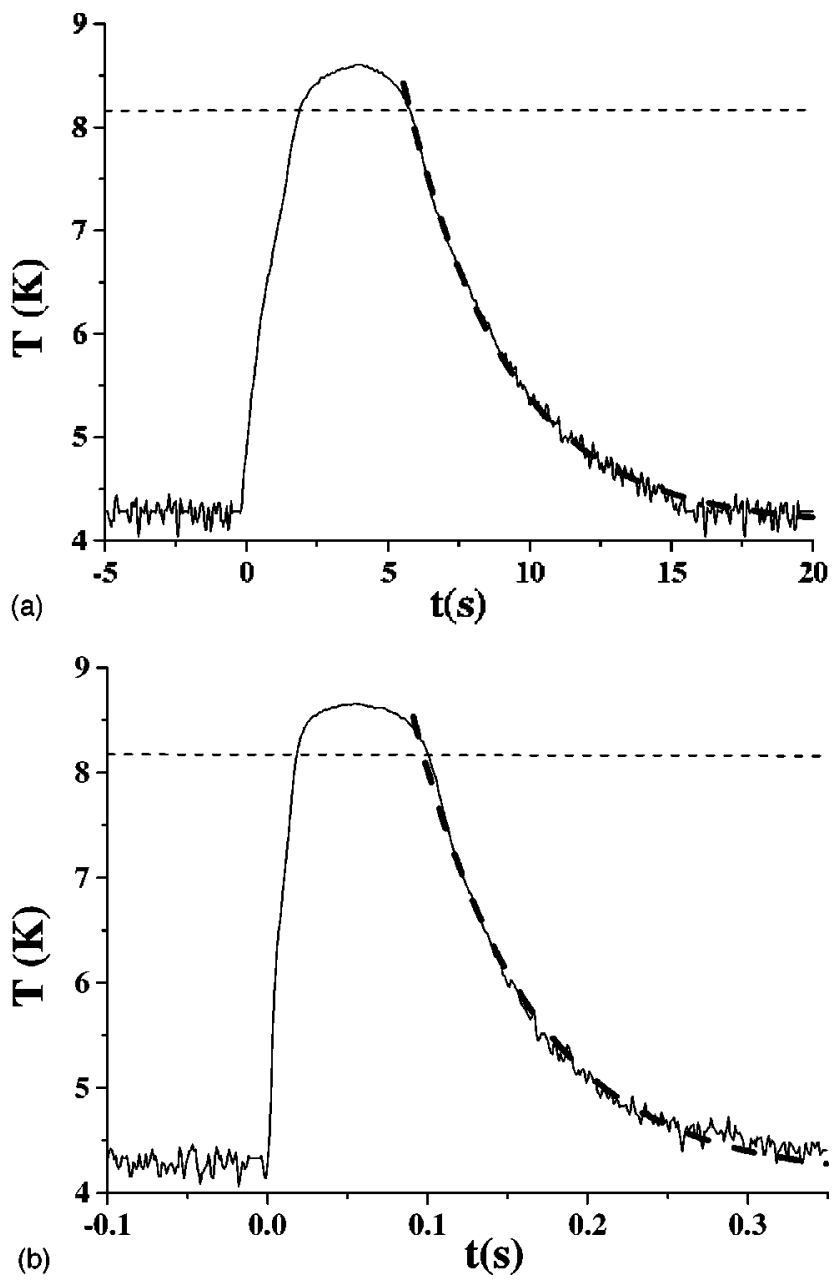

FIG. 4. Time dependence of the junction temperature during the (a) slow and (b) fast thermal cycle obtained from the data of Fig. 3 transformed according to Eq. (4). The horizontal dashed lines indicate the temperature threshold below which the temperature time dependence can be reliably accounted for by our measured data. Furthermore, the thick dashed lines are best-fitting curve of the cooling process according to the thermal relaxation expression Eq. (5).

racy. We would like to mention that an experimental proof of Eq. (4) in $\mathrm{Nb} / \mathrm{Nb}$ tunnel junctions was first evidenced by Broom. ${ }^{25}$

Figures 4(a) and 4(b) show the data reported in Figs. 3(a) and 3(b), respectively, transformed according to Eq. (4), assuming for $\Delta(0)$ and $T_{c}$ the values $2.85 \mathrm{meV}$ and $8.95 \mathrm{~K}$, respectively, as found by Monaco et al. ${ }^{26}$ on similar JTJ's. Now the dashed horizontal lines indicate the temperature threshold below which the temperature time dependence can be reliably accounted for by our measured data. The large noise at low temperature is the result of an amplification effect of Eq. (4), according to which the temperature variation corresponding to a given energy gap variation becomes larger and larger as the temperature becomes smaller. It would be very complicated to write the proper boundary conditions for the heat diffusion equation that would correctly model the full time dependence of the junction temperature. 


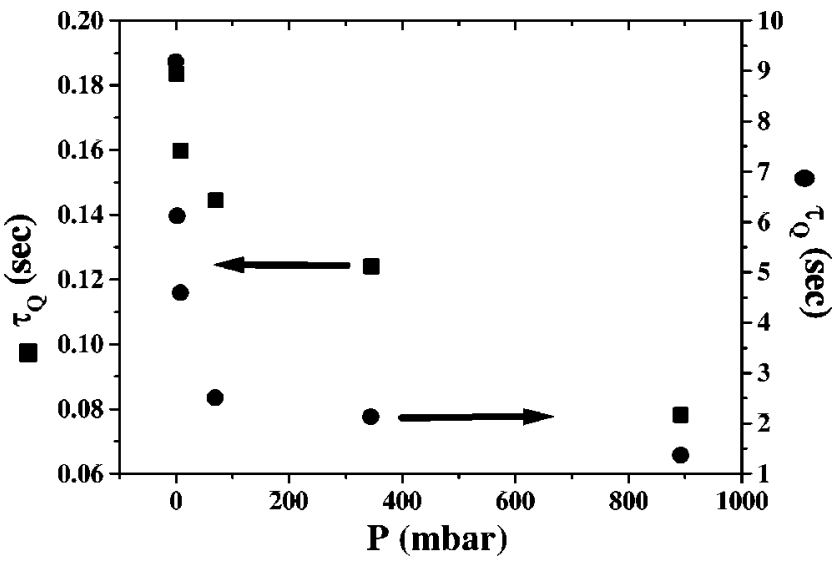

FIG. 5. Quenching time $\tau_{Q}$ as a function of the He gas pressure inside the vacuum tight can. By changing the pressure of the exchange gas inside the can, the system thermal constants are varied. The solid squares refer to the left vertical scale while the solid circles refers to the right vertical scale.

However, for our purposes we are only interested in the cooling process, and we successfully fit our data by a simple thermal relaxation equation:

$$
T(t)=T_{f i n}+\left(T_{i n}-T_{f i n}\right) \exp \left(-\frac{t-t_{0}}{\tau}\right)
$$

with only two fitting parameters $t_{0}$ and $\tau, T_{\text {in }}$ and $T_{\text {fin }}$ being fixed at 8.95 and $4.15 \mathrm{~K}$, respectively. In Eq. (5) $t_{0}$ is the time at which $T=T_{i n}=T_{c}$ and $\tau$ is the relaxation time that sets the cooling time scale. The fitting curves are shown by the thick dashed lines in Figs. 4(a) and 4(b), and correspond to a thermal relaxation time $\tau$ equal to 3.6 and $0.073 \mathrm{~s}$, respectively. The quenching time $\tau_{Q}$ can be obtained from its definition:

$$
\frac{T_{C}}{\tau_{Q}}=-\left.\frac{d T}{d t}\right|_{T=T_{C}}
$$

giving $\tau_{Q}=\tau T_{C} /\left(T_{i n}-T_{\text {fin }}\right)$. For a thermal relaxation from the junction critical temperature down to the helium bath temperature we get $\tau_{Q} \simeq 1.9 \tau$. Eventually, Fig. 5 displays the values of the quenching times obtained with the process described above, both for the fast (black squares with right vertical scale) and slow (solid circles with left vertical scale) cooling processes and for different values of the He pressure inside the can. We observe that, by changing the exchange gas pressure and using the two techniques, the quenching time can be changed over a quite large range starting from tenths to tens of seconds. At the end of this paper we will discuss how it is possible to extend this range in both directions; however, as we will show in the following section, this range has shown to be large enough for our purposes.

\section{THE MEASUREMENTS}

Quenching experiments were carried out in a double $\mu$-metal shielded cryostat and the transitions from the normal to the superconducting states were performed with no current flowing in the heaters and the thermometers. Both the junction voltage and current leads were shorted during all the thermal cycle. Furthermore, the heat supplied to the sample was such that the maximum temperature reached by the junction was made slightly larger than its critical temperature, say at about $10 \mathrm{~K}$, in order to make sure that also the bulk electrode critical temperature $\left(T_{C} \simeq 9.2 \mathrm{~K}\right)$ was overcome. In this case, according to Eqs. (5) and (6), the value of the quenching time results in a correspondingly smaller $\tau_{Q}$ $\simeq 1.7 \tau$. Due to the approximation made by using Eq. (5) and to the experimental uncertainty in the knowledge of the maximum temperature during each thermal cycle, the value of the quenching times has been determined with an overall accuracy as large as $5 \%$. For each value of the quenching time, in order to estimate the trapping probability, we have carried out a set of 300 thermal cycles and at the end of each cycle the junction CVC was inspected in order to ascertain the possible spontaneous trapping of one or more fluxons.

As we shall see later, the AJTJ's are such that the ZK causal length $\bar{\xi}>C$ by an order of magnitude when $\tau_{Q}$ $=1 \mathrm{~s}$. Increasing and decreasing $\tau_{Q}$ by an order of magnitude changes $\bar{\xi}$ by less than a factor of 2 . Thus the probability of finding a single fluxon after a quench is small. In the following, we will focus our attention only on the probability $P_{1}$ to trap just one fluxon, although a few times we found clear evidence of two and, more seldom, three homopolar fluxons spontaneously trapped during the $N-S$ transition. However, these events were too rare to be statistically significant.

Experimentally, we define $P_{1}$ as the ratio between the number of times in which, at the end of the thermal cycle, the junction CVC looks like that shown in Fig. 1(b), i.e., with a tiny critical current and a large first ZFS, and the number of attempts. It is worth mention here that in the case of simultaneous trapping of a fluxon and an antifluxon, they would annihilate and leave no track of their formation. Therefore, our definition of $P_{1}$ is not rigorous, but it is reasonable as far as the chances to trap two (homo or heteropolar) fluxons are negligibly small. Similarly, a CVC similar to that shown in Fig. 1(b) could be the result of the simultaneous trapping of two fluxons and one antifluxon, or the other way around, but this event is less likely, to occur in our experimental situation. For the sake of completeness, it must be added that, in some cases, the CVC displayed either a depressed critical current without ZFS structures or a ZFS with an enhanced critical current. We explain them as due to the trapping of Abrikosov vortices in the junction electrodes and nearby the barrier and we did not take in to account the occurrence of such events, since it is not known if and how the vicinity of Abrikosov vortices influences the fluxon formation.

\section{THE RESULTS}

When $\bar{\xi}>C$, we estimate the probability of finding a fluxon in a single quench to be

$$
P_{1} \simeq \frac{C}{\bar{\xi}}=\frac{C}{\xi_{0}}\left(\frac{\tau_{Q}}{\tau_{0}}\right)^{-\sigma},
$$




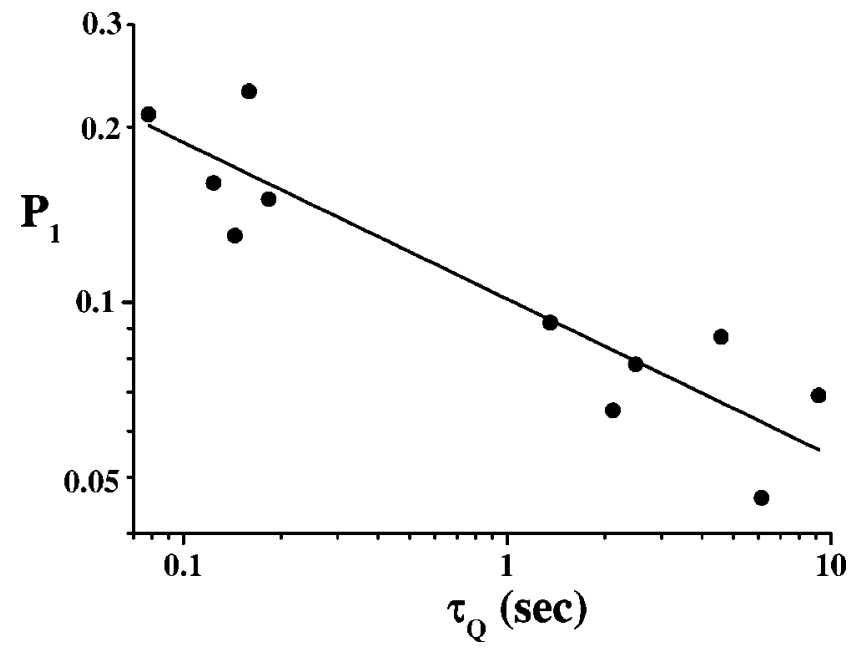

FIG. 6. Log-log plot of the measured probability $P_{1}$ to trap one fluxon versus the quenching time $\tau_{Q}$. The solid line is the best fitting curve found assuming a power-law dependence as suggested by Eq. (1). To a good degree of approximation, the fit is in agreement with a fourth root square dependence as expected for symmetric annular Josephson tunnel junctions.

where, from Eq. (3), $\sigma=0.25$.

Figure 6 shows on a log-log plot the measured probability $P_{1}$ of a single fluxon trapping obtained by quenching the sample $A 300$ times for each value of the quenching time $\tau_{Q}$ changed by varying the exchange gas pressure and by using both the fast and slow quenching techniques. We observe that the points are quite scattered, meaning that the data are statistically poor. Further, for the reasons given earlier, there is a gap between fast and slow quenches. Nonetheless, we have clear evidence that (i) the trapping of a fluxon occurs on a purely statistical basis, the conditions being identical for each thermal cycle and (ii) the probability to trap one fluxon is larger when the transition is performed at a faster speed (smaller quenching time) in a qualitative accordance with the causality principle. More precisely, we can distinguish the point to the lower right of the graph corresponding to the slow cycle process that gives in the average a probability to spontaneously trap a fluxon once every 14 attempts, and the cloud of data to the upper left corresponding to the impulsive junction heating and giving an average probability of one successful event every about six attempts. This suggests that possible temperature gradients induced by the SMR are not an important source of systematic error, since such gradients, with their slowly moving profiles, have a tendency to reduce defect production. $^{27}$

Regardless of the data spread, as suggested if Eq. (7) holds true, we attempted to fit the data with an allometric function $P_{1}=a \tau_{Q}^{-b}$ with $a$ and $b$ being free fitting parameters. We found that the best-fitting curve, shown by the solid line in Fig. 6, has a slope $b=0.27 \pm 0.05$. Such a value of $b$, although affected by a $20 \%$ uncertainty, is in good agreement with the fourth root square dependence expected for a symmetric junction.

For the coefficient $a$ we found the best-fitting value of $0.1 \pm 10 \%$ ( $\tau_{Q}$ in seconds). This is to be compared with the predicted value of $C \tau_{0}^{1 / 4} / \xi_{0}$. Sample $A$ had a circumference
$C=500 \mu \mathrm{m}$. Its effective superconductor thickness was $d_{s}$ $\approx 250 \mathrm{~nm}$. At the final temperature $T_{\text {fin }}=4.2 \mathrm{~K}$, the critical current density was $J_{c}\left(T_{\text {fin }}\right)=3050 \mathrm{~A} / \mathrm{cm}^{2}$ and the Josephson length was $\lambda_{J}\left(T_{\text {fin }}\right)=6.9 \mu \mathrm{m}$. From this, and $c_{0}$ given earlier, we infer that $\xi_{0} \approx 3.8 \mu \mathrm{m}$ and $\tau_{0} \approx 0.17 \mathrm{ps}$. This then gives $C \tau_{0}^{1 / 4} / \xi_{0} \approx 0.08 \mathrm{~s}^{1 / 4}$, in good agreement with the experimental value of $b$, given the fact that we only expect agreement in overall normalization to somewhat better than an order of magnitude level. At this level, such a result is immune to systematic error in one or the other of the measurement processes. After the problems (discussed below) of the experiments discussed in (Refs. 7-9) to find (reliable) defects at expected densities, if at all, our experiment shows that the $\mathrm{ZK}$ estimate remains sensible.

Similar measurements have been carried out for sample $B$. Although not in contradiction with Eq. (1), the results were affected by a data scattering even larger than that found for sample $A$ (shown in Fig. 6). This is due to a much smaller normalized length that, according to Eq. (7), translates in an expected probability $P_{1}$, for a given $\tau_{Q}$, about four times smaller (since $C \tau_{0}^{1 / 4} / \xi_{0} \approx 0.02 \mathrm{~s}^{1 / 4}$ for this sample), far too small to get statistically significant data in reasonable times considering that these measurements are both very time and LHe consuming. In order to have data comparable with those of sample $A$, sample $B$ would have required a quenching times $4^{4}$ times larger. However, the roughly measured probability $P_{1}$ of 1 fluxon every 50-100 attempts is in fairly good agreement with the expected value. This shows that our fluxons are not spurious by-products of the measurement mechanism.

\section{COMMENTS, FUTURE EXPERIMENTS, AND CONCLUSIONS}

We consider this experiment to give a strong confirmation of the Zurek-Kibble predictions. We said in the introductory section of this paper that condensed-matter experiments to test the $\mathrm{ZK}$ predictions had given mixed results, and it is interesting to put this experiment in that context.

Prior to our experiment, seven other experiments had been performed to test Eq. (1), five with fixed $\tau_{Q},{ }^{5-9}$ two with variable $\tau_{Q} \cdot{ }^{10,11}$ [In addition, the experiment cited earlier $^{15}$ on JTJ's by two of us (R.M. and J.M.) was compatible with Eq. (3), although it had not been performed with a test of Eq. (3) in mind. It was this that motivated the experiment described here.]

Of those experiments with fixed $\tau_{Q}$, two were experiments ${ }^{5,6}$ on superfluid ${ }^{3} \mathrm{He}-\mathrm{B}$, which rely on the fact that when it is bombarded with slow neutrons energy is released, which leads to a hot spot, with temperature $T>T_{c}$, in the superfluid that then cools below $T_{c}$. This leaves behind a tangle of vortices, the topological defects in this system, whose density can be measured. Since $\tau_{Q}$ is fixed by the nuclear process, it is not possible to confirm the predicted value $\sigma=1 / 4$. However, with only a single data point conflating both normalization and $\sigma$ both experiments are highly compatible with Eq. (1).

The remaining experiments with fixed $\tau_{Q}$ were two ${ }^{7,8}$ on 
superfluid ${ }^{4} \mathrm{He}$, and one ${ }^{9}$ on high-temperature superconductors (HTSC).

In principle, the ${ }^{4} \mathrm{He}$ experiments, ${ }^{7,8}$ which use a pressure quench with a varying time scale $\tau_{Q}$ to implement the transition, could have allowed for a more complete test, in this case to confirm $\sigma=1 / 3$ (after renormalization-group rescaling). Yet again, vortices are the relevant defects. In practice, the most reliable published experiment ${ }^{8}$ sees no vortices. In this context, the vortices seen in an earlier ${ }^{4} \mathrm{He}$ experiment, ${ }^{7}$ at levels compatible with Eq. (1), were most likely an artifact of the experimental setup. Further experiments on ${ }^{4} \mathrm{He}$ are underway.

The fifth experiment, ${ }^{9}$ on HTSC, measures total flux through a surface, i.e., the variance in the topological charge, carried in this case by the Abrikosov vortices. The vortex separation of Eq. (1) can be converted into a prediction for the variance, but no flux is seen in contradiction with this prediction, despite the phase separation that is a prerequisite for the result being seen elsewhere. ${ }^{28}$ There is no obvious explanation of this null result. An attempt to take gauge fields into account fully ${ }^{29}$ shows that there is an additional mechanism for vortex production in the thermal fluctuations of the magnetic field but, as yet, this seems insufficient to explain the result. Such a mechanism will not apply to the JTJ's considered in our experiment.

These early experiments have either provided one data point for Eq. (1), or have been null for whatever reason. Two subsequent experiments have permitted varying quench rates and so an estimate for $\sigma$. The most recent ${ }^{10}$ involves the Bénard-Marangoni conduction-convection transition, in which a homogeneous conduction state is broken into an hexagonal array of convection lines on heating. The defects here are not associated with the line zeros of an orderparameter field, and the viscosity-dependent $\sigma$ does not match the ZK prediction, most likely for that reason. The more relevant experiment ${ }^{11}$ is carried out in a nonlinear optical system, with a complex beam phase, i.e., the order parameter, satisfying a time-dependent Ginzburg-Landau equation with drift. There has been much numerical analysis ${ }^{30}$ of time-dependent Ginzburg-Landau systems, which show agreement with the $\mathrm{ZK}$ predictions for scaling exponents. The control parameter in (Ref. 11) is not the temperature, but the light intensity. Increasing it leads to pattern formation (defects) at a critical value. The predicted scaling parameter $\sigma=1 / 4$ is recovered to good accuracy as $\sigma_{\text {exp }}=0.25 \pm 0.02$, but agreement with normalization is not stated.

Given this relatively poor success rate in confirming Eq. (1) we are considering a further experiment to measure the
ZK scaling exponent, this time with manifestly nonsymmetric AJTJ's. In Ref. 13 and 14 we observed that it should have been difficult to make JTJ's truly symmetric, as those used here. However, in Ref. 13 and 14 we had not appreciated how the difference between symmetric and marginally nonsymmetric JTJ's is smeared by the proximity effect of $\mathrm{Al}$ within the insulating layer. Significantly nonsymmetric JTJ's require different fabrication techniques, but the value of $\sigma$ inferred from the same causal arguments is $\sigma=1 / 7$, which is very different from the value of $1 / 4$ that we tested above. The data from our experiment is incompatible with $\sigma=1 / 7$. This does suggest that a further experiment, with markedly nonsymmetric JTJ's, should be performed.

Our experiments have demonstrated that quenching time of the order of $1 \mathrm{~s}$ gives a rather large probability to trap one fluxons on AJTJ's having a very large normalized length. However, very long junctions mean very large critical current densities that, in turn, require Josephson barriers so thin that their quality and uniformity is often spoiled; furthermore, in most cases, applications require an intermediate length junction or even small junction. For these reasons, it would be highly desirable to compensate the reduced junction length with an increased quenching rate, as it is suggested by the findings for sample $B$. Therefore, we like to conclude the paper with some comments on the possible technical improvements that would allow one to test the $\mathrm{ZK}$ predictions over a broader quenching time range. First, $\tau_{Q}$ can be trivially heightened by increasing the $\mathrm{Cu}$ block thermal capacitance. On the contrary, in order to lower the quenching time, that is to make the $N-S$ transition faster, it is needed to resort to new techniques since the maximum power that can be dissipated by the surface mount resistors sets an obvious lower threshold on $\tau_{Q}$. One possible way to reach this goal is to perform the junction thermal cycle by means of light pulses. Light dissipates inside the superconducting electrodes, but not in the substrate providing a local junction heating that will relax much faster to the background temperature. We estimate that, by using a properly focused pulsed light beam, the quenching time scale can be reduced to the microsecond range.

\section{ACKNOWLEDGMENTS}

The authors thank L. Filippenko for the sample fabrication and V.P. Koshelets for useful discussions. R.R. thanks the University of Salerno for hospitality. R.M. thanks the Technical University of Denmark for hospitality. This work was also supported by the COSLAB program of the European Science Foundation and the Hartmann Foundation.

\footnotetext{
*Email address: roberto@sa.infn.it

†Email address: myg@fysik.dtu.dk

${ }^{\ddagger}$ Permanent address: Imperial College, London, SW7 2BZ, U.K. Email address: r.rivers@ic.ac.uk

${ }^{1}$ T.W.B. Kibble, J. Phys. A 9, 1387 (1976).

${ }^{2}$ T.W.B. Kibble, Phys. Rep. 67, 183 (1980).

${ }^{3}$ W.H. Zurek, Nature (London) 317, 505 (1985); Acta Phys. Pol. B 24, 1301 (1993).

${ }^{4}$ W.H. Zurek, Phys. Rep. 276, 177 (1996).
}

${ }^{5}$ V.M.H. Ruutu, V.B. Eltsov, A.J. Gill, T.W.B. Kibble, M. Krusius, Y.G. Makhlin, B. Placais, and G.E. Volovik, Nature (London) 382, 334 (1996).

${ }^{6}$ C. Bauerle, Y.M. Bunkov, S.N. Fisher, H. Godfrin, and G.R. Pickett, Nature (London) 382, 332 (1996).

${ }^{7}$ P.C. Hendry, N.S. Lawson, R.A.M. Lee, P.V.E. McClintock, and C.D.H. Williams, Nature (London) 368, 315 (1994).

${ }^{8}$ M.E. Dodd, P.C. Hendry, N.S. Lawson, P.V.E. McClintock, and C.D.H. Williams, Phys. Rev. Lett. 81, 3703 (1998); J. Low 
Temp. Phys. 15, 89 (1999).

${ }^{9}$ R. Carmi and E. Polturak, Phys. Rev. B 60, 7595 (1999).

${ }^{10}$ S. Casado, W. González-Viñas, H. Mancini, and S. Boccaletti, Phys. Rev. E 63, 057301 (2001).

${ }^{11}$ S. Ducci, P.L. Ramazza, W. González-Viñas, and F.T. Arecchi, Phys. Rev. Lett. 83, 5210 (1999).

${ }^{12}$ R. Monaco, J. Mygind, and R.J. Rivers, Phys. Rev. Lett. 89, 080603 (2002).

${ }^{13}$ E. Kavoussanaki, R. Monaco, and R.J. Rivers, Phys. Rev. Lett. 85, 3452 (2000).

${ }^{14}$ R. Monaco, R.J. Rivers, and E. Kavoussanaki, J. Low Temp. Phys. 124, 85 (2001).

${ }^{15}$ N. Martucciello, J. Mygind, V.P. Koshelets, A.V. Shchukin, L.V. Filippenko, and R. Monaco, Phys. Rev. B 57, 5444 (1998).

${ }^{16}$ J.C. Swihart, J. Appl. Phys. 32, 461 (1961).

${ }^{17}$ E. Kavoussanaki, R.J. Rivers, and G. Karra, Condens. Matter Phys. 3, 133 (2000).

${ }^{18}$ R.J. Rivers, J. Low Temp. Phys. 124, 41 (2001).

${ }^{19}$ N. Grønbech-Jensen, P.S. Lomdahl, and M.R. Samuelsen, Phys. Lett. A 154, 14 (1991).
${ }^{20}$ D.W. McLaughlin and A.C. Scott, Phys. Rev. A 18, 1652 (1978).

${ }^{21}$ N. Martucciello and R. Monaco, Phys. Rev. B 53, 3471 (1996).

${ }^{22}$ A. Barone and G. Paterno, Physics and Applications of the Josephson Effect (Wiley, New York 1982); V. Ambegaokar and A. Baratoff, Phys. Rev. Lett. 10, 486 (1963); 11, 104(E) (1963).

${ }^{23}$ L.V. Filippenko, S.V. Shitov, P.N. Dmitriev, A.B. Ermakov, V.P. Koshelets, and J.R. Gao, IEEE Trans. Appl. Supercond. 11 (1), 816 (2001).

${ }^{24}$ D.J. Thouless, Phys. Rev. 117, 1256 (1960).

${ }^{25}$ R.F. Broom, J. Appl. Phys. 47, 5432 (1976).

${ }^{26}$ R. Monaco, R. Cristiano, L. Frunzio, and C. Nappi, J. Appl. Phys. 71, 1888 (1992).

${ }^{27}$ T.W.B. Kibble and G.E. Volovik, JETP Lett. 65, 102 (1997).

${ }^{28}$ R. Carmi, E. Polturak, and G. Koren, Phys. Rev. Lett. 84, 4966 (2000).

${ }^{29}$ M. Hindmarsh and A. Rajantie, Phys. Rev. Lett. 85, 4660 (2000); A. Rajantie, J. Low Temp. Phys. 124, 5 (2001).

${ }^{30}$ P. Laguna and W.H. Zurek, Phys. Rev. Lett. 78, 2519 (1997); A. Yates and W.H. Zurek, ibid. 80, 5477 (1998); N.D. Antunes, L.M.A. Bettencourt, and W.H. Zurek, ibid. 82, 2824 (1999). 